OPEN ACCESS

Edited by:

Zhen Shen,

Nanjing University, China

Reviewed by:

Hua Lu,

Hangzhou Normal University, China Jiangli Fan,

Dalian University of Technology, China John Mack,

Rhodes University, South Africa

*Correspondence: Lijuan Jiao

jiao421@ahnu.edu.cn

Specialty section: This article was submitted to

Supramolecular Chemistry,

a section of the journal

Frontiers in Chemistry

Received: 31 July 2019 Accepted: 13 November 2019 Published: 26 November 2019

Citation:

Miao W, Yu C, Hao E and Jiao L (2019) Functionalized BODIPYs as

Fluorescent Molecular Rotors for Viscosity Detection

Front. Chem. 7:825

doi: 10.3389/fchem.2019.00825

\section{Functionalized BODIPYs as Fluorescent Molecular Rotors for Viscosity Detection}

\author{
Wei Miao, Changjiang Yu, Erhong Hao and Lijuan Jiao* \\ The Key Laboratory of Functional Molecular Solids, Ministry of Education, Anhui Laboratory of Molecule-Based Materials, \\ School of Chemistry and Materials Science, Anhui Normal University, Wuhu, China
}

Abnormal changes of intracellular microviscosity are associated with a series of pathologies and diseases. Therefore, monitoring viscosity at cellular and subcellular levels is important for pathological research. Fluorescent molecular rotors (FMRs) have recently been developed to detect viscosity through a linear correlation between fluorescence intensity or lifetime and viscosity. Recently, 4,4-difluoro-4-bora-3a,4a-diaza-s-indacene (boron dipyrrins or BODIPY) derivatives have been widely used to build FMRs for viscosity probes due to their high rotational ability of the rotor and potentially high brightness. In this minireview, functionalized BODIPYs as FMRs for viscosity detection were collected, analyzed and summarized.

Keywords: viscosity, BODIPY, fluorescent molecular rotor, viscosimeter, fluorescent probe, dyes

\section{INTRODUCTION}

The viscosity of cells is an important parameter of the cellular microenvironment, which influences the interaction and transport of biological molecules and signals in living cells (Minton, 2001). Abnormal changes of intracellular microviscosity are associated with a series of pathologies and diseases (Nadiv et al., 1994). Thus, the development of suitable imaging tools to monitor and detect cellular microviscosity is important to study cellular function in both health and disease. Fluorescent molecular rotors (FMRs) are established as tools for monitoring cellular and subcellular viscosity changes because of their high sensitivity, fast-response and non-invasive testing of targets in biological systems. A common feature of FMR is that it consists of two moieties, which are connected by a single bond. One moiety with a large moment of inertia is considered to be fixed, called the stator, and the other moiety with a smaller moment of inertia is called the rotor (Figure 1A). In a low viscosity medium, the rotor rotates freely, and the energy of excitation is dissipated with non-radiative energy. However, in a high viscosity medium, rotation through the C$\mathrm{C}$ bond is constrained, and the excitation energy is released as emission with enhanced fluorescence intensity and lifetime (Uzhinov et al., 2011; Lee et al., 2018). Therefore, the physical mechanism of viscosity dependence of fluorescence quantum yield and lifetime is caused by the steric hindrance of intramolecular rotation. Recently, FMRs have been widely used to measure viscosity of local environment using their changes in fluorescence intensity and lifetime (Kuimova, 2012). From a practical point of view, FMR with high extinction coefficients, long (NIR) excitation wavelength, and potentially high brightness would be desirable (Ning et al., 2017; Hou et al., 2018). 
A

\section{Fluorescent Molecular Rotors}

C<smiles>CCOc1ccc(C(c2ccc[nH]2)c2ccc[nH]2)cc1</smiles>

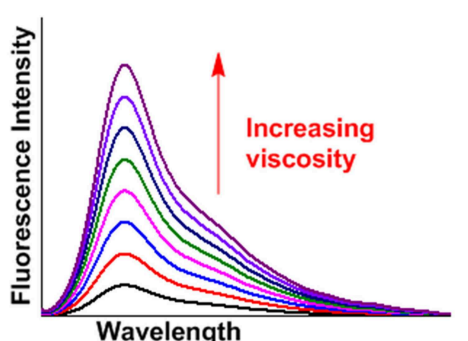

Wavelength
B

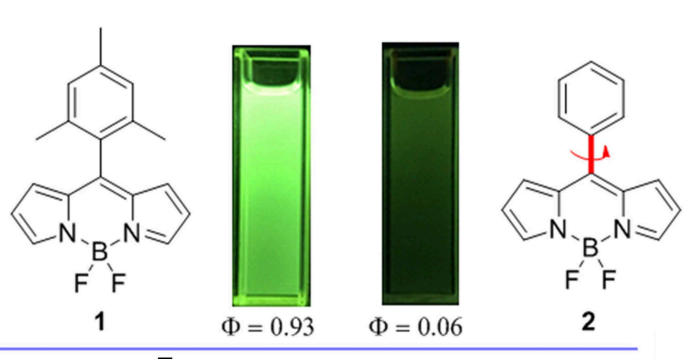

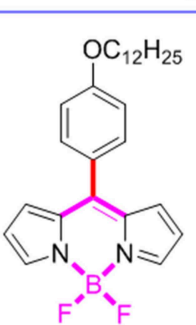

3

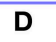

4a $\mathrm{R}_{1}=\mathrm{H}, \mathrm{R}_{2}=\mathrm{CH}_{3}$ 4b $\mathrm{R}_{1}=\mathrm{R}_{2}=\mathrm{CH}_{3}$
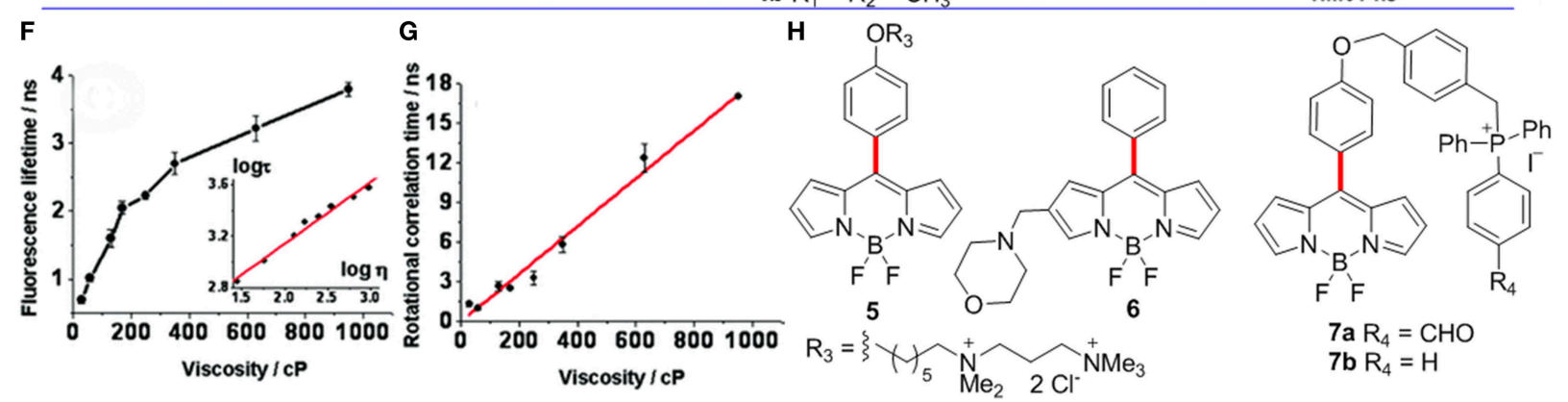

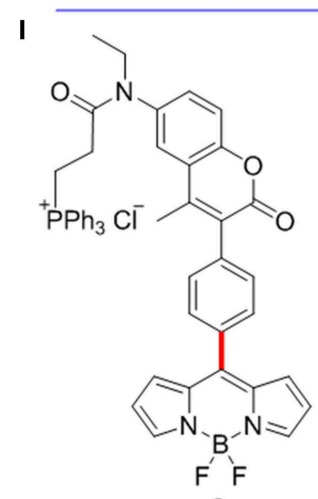

8

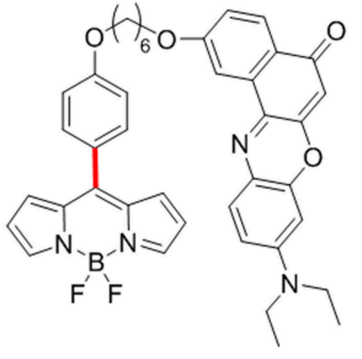

9

J

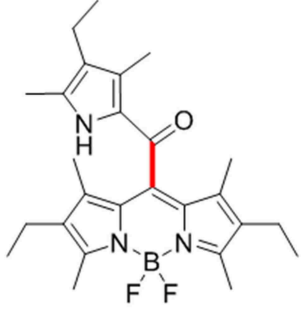

10

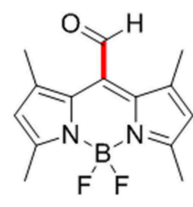

11

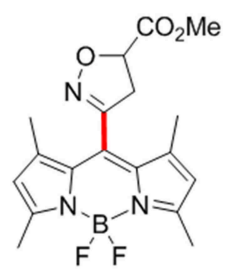

12

FIGURE 1 | (A) The working principle of FMRs. (B) Pictures of BODIPYs 1-2 in dichloromethane under $365 \mathrm{~nm}$ UV-light irradiation. (C) Synthetic route for meso-functionalized BODIPY 3. (D) Meso-functionalized BODIPYs 4. (E-G) Fluorescence lifetime and rotational correlation time recorded for BODIPY $\mathbf{3}$ in solvents of various viscosities. Reproduced with permission from Kuimova et al. (2008), Copyright 2008 American Chemical Society. (H-J) Meso-functionalized BODIPYs 5-12 as FMRs.

Recently, 4,4-difluoro-4-bora-3a,4a-diaza-s-indacenes (BODIPYs) and their derivatives have been paid much attention because of their excellent chemical and physical properties (Loudet and Burgess, 2007; Lu et al., 2014), such as easy functionalization, high molar extinction coefficients, tunable visible to red excitation wavelength, tunable fluorescence quantum yields, as well as excellent photostabilities (Miao et al., 2019; Wang et al., 2019). Not surprisingly, BODIPY derivatives are widely used as imaging probes, fluorescent organic devices, chemical sensors, and as photosensitizers (Cui et al., 2014, 2015; Peterson et al., 2018; Turksoy et al., 2019).

The fluorescence quantum yield of meso-(2,4,6trimethylphenyl)BODIPY $\mathbf{1}$ is 0.93 in toluene. However, the fluorescence quantum yield of meso-phenylBODIPY $\mathbf{2}$ is only 0.06 in toluene (Figure 1B; Kee et al., 2005). The difference results from free rotations of the meso-phenyl group of BODIPY 
2 causing energy dissipations as non-radiative. The rotation of its meso-phenyl group is constrained by increasing the viscosity of local environment around BODIPY 2. Therefore, meso-phenylBODIPY $\mathbf{2}$ has the potential to be used as a FMR for viscosity detection. Subsequently, various BODIPY derivatives have indeed been developed as FMRs for viscosity sensors, and the rotor-moiety is mainly attached at the meso-position and 2,6-position of the BODIPY scaffold (Dziuba et al., 2016). In this minireview, functionalized BODIPYs as FMRs for viscosity detection are collected, analyzed, and summarized.

\section{Meso-Functionalized BODIPYs as FMRs}

In biological systems, changes in viscosity at the cellular level are associated with diseases and pathologies, such as diabetes, infarction, and hypertension. Initially, FMRs were designed to detect cellular viscosity through fluorescence intensity (Haidekker and Theodorakis, 2007). However, measurements based on fluorescence intensity are affected by intracellular uncertainty concentrations. To overcome the problem, Kuimova et al. reported a first meso-functionalized BODIPY 3 as a FMR, which detects intracellular viscosity by using fluorescence lifetime instead of fluorescence intensity (Kuimova et al., 2008). As shown in Figure 1C, BODIPY 3 was synthesized through oxidation of the corresponding dipyrromethane with DDQ followed by addition of excess amounts of base and $\mathrm{BF}_{3}$. OEt 2 . The authors measured the fluorescence of BODIPY 3 at various viscosities, and indicated that both the fluorescence quantum yield and lifetime (from $0.7 \pm 0.05$ to $3.8 \pm 0.1$ ns; Figure 1E) increased dramatically when increasing of the viscosity between 28 and $950 \mathrm{cP}$. Importantly, the plot of $\log \tau$ ( $\tau$ is fluorescence lifetime) vs. $\log \eta$ ( $\eta$ is viscosity) was fitted by a straight line (Figure 1F). Control BODIPYs 4a-b (Figure 1D) were also studied. Since free rotations of the phenyl groups of BODIPYs $\mathbf{4 a - b}$ are restricted, the nonradiative decay process in both dyes is thus prevented. As expected, BODIPYs 4a-b have fluorescent quantum yields of close to unity in solvents. No apparent viscosity induced fluorescent intensity or lifetime was observed for both $\mathbf{4 a}$ and 4b. Subsequently, the fluorescence lifetime imaging (FLIM) using 3 was carried out to study intracellular viscosity, and the results indicated that the average viscosity of SK-OV-3 cells was $140 \pm 40 \mathrm{cP}$. Time-resolved fluorescence anisotropy decays with various viscosities were recorded to confirm that the above high viscosity value didn't result from the binding of the rotor to the intracellular targets. The results showed that the rotational correlation time $(\theta)$ of BODIPY 3 increased linearly with solvent viscosity (Figure 1G). Finally, the viscosity in SK-OV-3 cells was also measured by using polarizationresolved time correlated single photon counting (TCSPC), and found that the average viscosity of SK-OV-3 cells is $80 \mathrm{cP}$ according to a linear relationship between rotational correlation time $\theta$ and viscosity $\eta$. The average viscosity of SK-OV-3 cells measured by the two methods is compatible, which shows that the rotor does not combine to intracellular targets. Therefore, the FLIM method using BODIPY based on FMRs is a versatile and also practical method for detecting intracellular viscosity.
Viscosity changes in membranes are associated with various intracellular physiological processes, particularly with various diseases. A few FMRs have successfully detected the viscosity of model lipid bilayers (Hosny et al., 2013; Wu et al., 2013). However, it is difficult to detect viscosity of plasma membranes because of possible effective endocytosis of the probe. For example, BODIPY 3 was only reported to detect viscosity of the lipid membranes of internal cellular organelles. LópezDuarte et al. reported a meso-functionalized BODIPY 5 to selectively detect viscosity of plasma membranes by adding a double positive charge to the hydrocarbon tail of BODIPY 3 (López-Duarte et al., 2014), which could prevent enucleation and maintain rotor function at the same time (Figure $\mathbf{1 H}$ ). The fluorescence intensity and lifetime measurements of rotor 5 were studied in solvents of various viscosities. As expected, the gradual increase of the viscosity from 0.6 to $930 \mathrm{cP}$ gave a continuous enhancement of both its fluorescence emission intensity and lifetime. In order to illustrate that this dye is mainly distributed on the cell membrane, the uptake experiments of $5(8.9 \mu \mathrm{M})$ were carried out at $4^{\circ} \mathrm{C}$ with $\mathrm{Mg}^{2+}$ and $\mathrm{Ca}^{2+}$ free medium in SK-OV-3 cells. The colocalization results indicated 5 exclusively stained the plasma membranes of SK-OV-3 cells after incubation for more than $30 \mathrm{~min}$. The internal staining of the cells became obvious after incubation time of $55 \mathrm{~min}$. In addition, the FLIM of 5, measured in SK-OV-3 cells, showed that some staining of internal organelles for an incubation time of $40 \mathrm{~min}$. The lifetimes obtained from this lifetime histogram (40 min) were lower than that obtained after only $10 \mathrm{~min}$ incubation. The lifetime histogram for the $40 \mathrm{~min}$ image is more adequately fitted with a bimodal Gaussian peak fit, and individual peaks are centered at $1.9 \mathrm{~ns}$ (organelles, $200 \mathrm{cP}$ ) and $2.2 \mathrm{~ns}$ (plasma membrane, $270 \mathrm{cP}$ ).

The lysosomal viscosity reflects the microscopic state and function of this organelle. When lysosomal function is impaired, especially through lysosomal storage disease caused by single lysosomal enzymes deficiency, macromolecular substances cannot be decomposed and accumulate in lysozyme. Therefore, it is important to monitor the changes of lysosomal viscosity in real time. In this respect, Wang et al. reported BODIPY 6 with a morpholine moiety at the 2-position of meso-phenylBODIPY as a FMR to detected viscosity of lysosome by using the FLIM method (Figure 1H; Wang et al., 2013). Morpholine unit was used as an excellent lysosomal localization group according to previous reports (Yu et al., 2012). Indeed, colocalization experiments showed that $\mathbf{6}$ can selectively strain cell lysosome. At first, they measured the fluorescence intensity of $\mathbf{6}$ in different $\mathrm{pH}$ at a particular viscosity, and revealed that fluorescence intensity of $\mathbf{6}$ was disturbed by $\mathrm{pH}$ changes. In contrast, the lifetimes of $\mathbf{6}$ at different $\mathrm{pH}$ were very similar. Next, they measured fluorescence lifetime of $\mathbf{6}$ in a series of buffers with different viscosities (from 0.6 to $359.6 \mathrm{cP}$ ), and the results showed that its fluorescence lifetimes $(\log \tau)$ have a strong linear relationship with viscosities $(\log \eta)$. Subsequently, the FLIM of $\mathbf{6}$ was recorded in MCF-7 cells, and suggested that the average viscosity was $\sim 65 \mathrm{cP}$ in lysosome of MCF-7 cells according to the linear relationship of lifetime against viscosity. Finally, the FLIM of $\mathbf{6}$ was successfully monitored the dynamic changes of lysosomal 


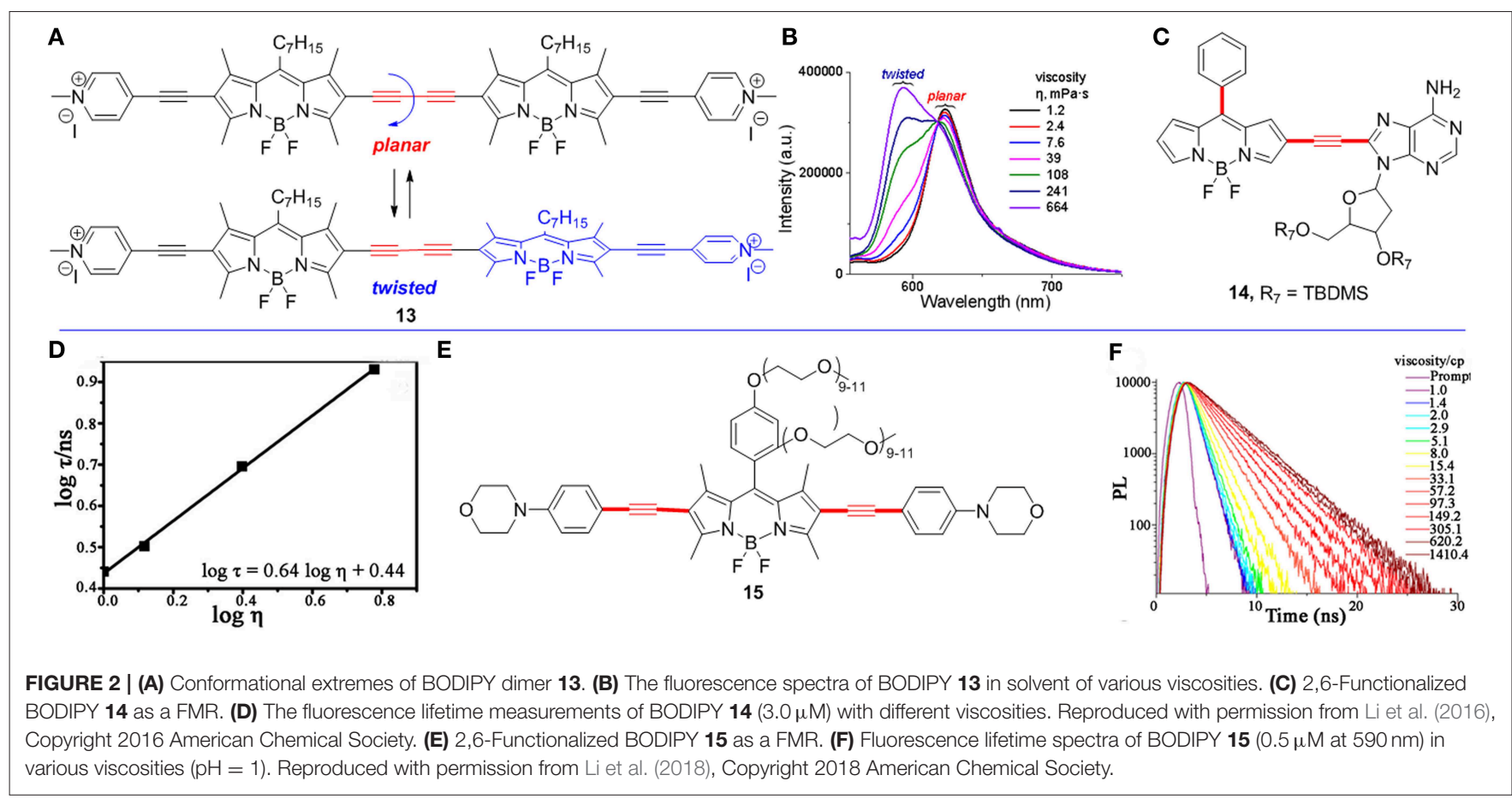

viscosity in both dexamethasone and chloroquine stimulated MCF-7 cells.

Mitochondria, as a membrane-bound subcellular organelle, have been found in almost all eukaryotic cells. Mitochondrial viscosity deviations from normal levels will affect the respiratory state of mitochondria, and induce cell dysfunction or even death. Song et al. reported BODIPY $7 \mathbf{a}$ as a novel fixable sensor for detecting mitochondrial viscosity of living cells by the FLIM method (Song et al., 2017). Initially, they synthesized two mitochondrial-localized BODIPYs $7 \mathbf{a}$ and $7 \mathbf{b}$ as showed in Figure 1H. Fluorescence lifetimes $(\log \tau)$ of both dyes have excellent linear relationships with viscosities $(\log \eta)$ from 0.6 to $360 \mathrm{cP}$. Moreover, colocalization studies confirmed that both $7 \mathbf{a}$ (Pearson's coefficient 0.92) and 7b (Pearson's coefficient 0.97) can specially localized in the mitochondria of the SMMC7721 cells. Another set of colocalization imaging experiments of $7 \mathbf{a}$ and 7b with Mito Tracker Deep Red were recorded under extreme condition ( $4 \%$ formaldehyde solution treatment). Interestingly, 7a exhibited strong intracellular fluorescence before and after the formaldehyde treatment. In contrast, $\mathbf{7 b}$ only showed strong fluorescence before formaldehyde treatment, however, its fluorescence was remarkably decreased after formaldehyde treatment. These data showed that BODIPY 7a was immobilized in the mitochondria. Next, the mitochondrial FLIM of BODIPYs 7a-b suggested that the average viscosity around BODIPYs 7ab in the mitochondria of the SMMC7721 cells is 95 and 63 $\mathrm{cP}$, respectively. Similar results were also found in other types of cells, e.g., MCF-7 cells. The authors explained that these different values are due to the different locations of probes: $7 \mathbf{a}$ is mainly immobilized on proteins of the mitochondria, while $\mathbf{7 b}$ is freely distributed in the mitochondria. The larger viscosity value measured by 7a may be the contribution of the macromolecule of the protein. Finally, the authors further monitored the viscosity changes in abnormal mitochondria (stimulated with rotenone) using BODIPY 7a. The fluorescence lifetime of BODIPY 7a was increased from 2.0 to 2.45 ns after stimulated for $8.5 \mathrm{~h}$, and further increased to $2.73 \mathrm{~ns}$ after stimulated for $18 \mathrm{~h}$. Thus, 7a, as a fixable and mitochondria selective FMR, shows a great potential for monitoring mitochondrial viscosity in real time (Zhang et al., 2019).

The second approach for quantitatively determining viscosity through FMRs is based on ratiometric fluorescence measurements. The ratiometric fluorescence probes have a self-calibration effect, which overcomes the uncertainty associated photobleaching, microenvironments, and local probe concentration for conventional fluorescence probes based on intensity changes. Yang et al. reported a fluorescence ratiometry viscosity probe $\mathbf{8}$ containing a coumarin unit, a BODIPY unit, and a mitochondria selective triphenylphosphonium group (Figure 1I; Yang et al., 2013). The fluorescence changes of 8 in a series of viscosities (from 0.59 to $945.35 \mathrm{cP}$ ) revealed that the emission intensities at $427 \mathrm{~nm}$ (the emission of the coumarin moiety) and $516 \mathrm{~nm}$ (the emission of the BODIPY moiety) both increased with increased viscosity. A strong linear relationship between fluorescence intensity ratio $\left(I_{516} / I_{427}\right)$ and viscosity $(\eta)$ was obtained. In addition, fluorescence lifetimes $(\log \tau)$ also have a good linear relationship with viscosities $(\log \eta)$. According to the above linear relationships, the mitochondrial viscosity in HeLa cells is 62.8 and $67.5 \mathrm{cP}$, respectively, by using both fluorescence ratiometry and FLIM. Using similar strategy, Yang et al. also reported BODIPY 9 to detect microviscosity of the endoplasmic reticulum by using both fluorescence 
ratiometry and the FLIM method (Figure 1I; Yang et al., 2014).

The BODIPY based FMRs described above all contain rotable meso-phenyl groups, while BODIPY $\mathbf{4 b}$ (Figure $\mathbf{1}$ ) containing substituents on the 1,7-positions of the BODIPY fluorophore is not suitable to be used as FMRs because the meso-aromatic group is restricted by the substituents on the 1,7-positions. Thus, it is possible that a less bulky group (than phenyl) on meso-position of BODIPY with substituents on the 1,7-positions may allow the rotation in non-viscous media and thus might provide an alternative strategy for designing BODIPY based efficient microviscosity probes. Indeed, $\mathrm{Yu}$ et al. recently reported a meso2-ketopyrrolyl-derived BODIPY $\mathbf{1 0}$ as a new FMR containing substituents on the 1,7-positions (Figure 1J; Yu et al., 2019). The fluorescence lifetime $(\log \tau)$ of BODIPY $\mathbf{1 0}$ has a linear relationship with viscosity $(\log \eta)$. Subsequently, this probe was used to detect viscosity changes during the pathological processes using the FLIM in MCF-7 cells. Moreover, a "distortedBODIPY"-based viscosity probe 11 with meso-CHO group was reported by Zhu et al. (Figure 1J; Zhu et al., 2014). Zatsikha et al. reported a five-membered ring substituted BODIPY 12, in which the fluorescence intensity $(\log I)$ has a linear relationship with viscosity $(\log \eta)$ (Figure 1J; Zatsikha et al., 2019).

\section{2,6-Functionalized BODIPYs as FMRs}

In comparison with the meso-functionalized BODIPYs, only a few 2,6-functionalized BODIPYs have been reported to be used as FMRs to detect viscosity. They typically built through the alkyne bridged rotor and BODIPY core. Recently, Zhang et al. reported a ratiometric fluorescence probe $\mathbf{1 3}$ with the two BODIPY units linked by butadiyne group (Figure 2A; Zhang et al., 2017). The fluorescence spectra of 13 at different viscosities (from 1.2 to 664 mPa.s) were measured, and its fluorescent emission maximum peaks gradually shifted from 624 to $593 \mathrm{~nm}$ with the increase of viscosities (Figure 2B). These two fluorescence emission peaks may be contributed by two extreme conformers of $\mathbf{1 3}$ with planar or twisted orientations of the two BODIPY units.

Li et al. reported a RNA-targeted BODIPY 14 as a new FMR to detect intracellular viscosity (Figure 2C; Li et al., 2016). FMR 14 showed two different maximum wavelengths at 496 and $565 \mathrm{~nm}$, respectively. Similar to most fluorescent ratiometry probes, fluorescence measurements of BODIPY 14 showed two different emitted wavelengths (496 and $565 \mathrm{~nm}$ ), and both fluorescence intensity $\left(\log \mathrm{F}_{565} / \mathrm{F}_{496}\right)$ and lifetime $(\log \tau)$ have a linear relationship with and viscosity $(\log \eta)$ (Figure 2D). In addition, the colocalization experiments of BODIPY 14 and commercial RNA dye revealed that BODIPY $\mathbf{1 4}$ mainly distributed in cytoplasmic RNA (Pearson's correlation 0.96). This result was further supported by imaging of BODIPY $\mathbf{1 4}$ in four blood cell types. There was strong fluorescence in reticulocytes (contain RNA), but no fluorescence in red blood cells and other cells (without RNA).

Another FMR 15 (Figure 2E; Li et al., 2018) with two morpholine moieties selectively detected lysosomal viscosity using FLIM. Similar to BODIPY 6, the fluorescence lifetime $(\log \tau)$ of BODIPY 15 linearly increased with the increased viscosity (Figure $2 \mathrm{~F}, \mathrm{pH}=1$ ). Moreover, the colocalization experiments indicated that BODIPY $\mathbf{1 5}$ mainly distributed in lysosome in Hela cells (Pearson's coefficient 0.95). Next, they monitored the viscosity changes in abnormal mitochondria using BODIPY 15 by treating Hela cells with dexamethasone. Without treating dexamethasone, the histogram of BODIPY 15 indicated the viscosity of the lysosome is $15 \mathrm{cP}$ in Hela cells. However, the viscosity of the lysosome becomes $159 \mathrm{cP}$ after treating with dexamethasone for $1 \mathrm{~h}$ according to the linear relationship of lifetime-viscosity.

\section{CONCLUSION}

In summary, functionalized BODIPYs have recently been developed as novel FMRs for viscosity detection by fluorescence intensity and fluorescence lifetime, in which the rotormoieties are mainly attached at the meso-position and 2/6-positions of the BODIPY scaffold. Those BODIPY based FMRs can be used to detect the subcellular viscosity by introducing a localization group, such as a pyridinium salt, triphenylphosphine salt or morpholine, through fluorescence ratiometry and FLIM methods. By taking advantage of the rapid development of BODIPY synthesis and postfunctionalization, we can anticipate that more exciting BODIPY based FMRs decorated with various functional groups with red to near infrared absorption and emission will be developed. BODIPY based FMRs with rotation around other positions (B position, especially) will also be highly anticipated.

\section{AUTHOR CONTRIBUTIONS}

All authors listed have made a substantial, direct and intellectual contribution to the work, and approved it for publication.

\section{ACKNOWLEDGMENTS}

We thank the National Nature Science Foundation of China (Grants Nos. 21672006, 21672007, and 21871006) for supporting this work.

\section{REFERENCES}

Cui, X., Zhang, X., Xu, K., and Zhao, J. (2015). Application of singlet energy transfer in triplet state formation: broadband visible light-absorbing triplet photosensitizers, molecular structure design, related photophysics and applications. J. Mater. Chem. C 3, 8735-8759. doi: 10.1039/C5TC01401H

Cui, X., Zhao, J., Zhou, Y., Ma, J., and Zhao, Y. (2014). Reversible photoswitching of triplet-triplet annihilation upconversion using dithienylethene photochromic switches. J. Am. Chem. Soc. 136, 9256-9259. doi: 10.1021/ja504211y

Dziuba, D., Jurkiewicz, P., Cebecauer, M., Hof, M., and Hocek, M. (2016). A rotational BODIPY nucleotide: an environment-sensitive fluorescence-lifetime 
probe for DNA interactions and applications in live-cell microscopy. Angew. Chem. Int. Ed. 55, 174-178. doi: 10.1002/anie.201507922

Haidekker, M. A., and Theodorakis, E. A. (2007). Molecular rotors-fluorescent biosensors for viscosity and flow. Org. Biomol. Chem. 5, 1669-1678. doi: 10.1039/B618415D

Hosny, N. A., Mohamedi, G., Rademeyer, P., Owen, J., Wu, Y., Tang, M.X., et al. (2013). Mapping microbubble viscosity using fluorescence lifetime imaging of molecular rotors. Proc. Natl. Acad. Sci. U.S.A. 110, 9225-9230. doi: $10.1073 /$ pnas.1301479110

Hou, L., Ning, P., Feng, Y., Ding, Y., Bai, L., Li, L., et al. (2018). Two-photon fluorescent probe for monitoring autophagy via fluorescence lifetime imaging. Anal. Chem. 90, 7122-7126. doi: 10.1021/acs.analchem.8b01631

Kee, H. L., Kirmaier, C., Yu, L., Thamyongkit, P., Youngblood, W. J., Calder, M. E., et al. (2005). Structural control of the photodynamics of boron-dipyrrin complexes. J. Phys. Chem. B 109, 20433-20443. doi: 10.1021/jp0525078

Kuimova, M. K. (2012). Molecular rotors image intracellular viscosity. Chimia 66, 159-165. doi: 10.2533/chimia.2012.159

Kuimova, M. K., Yahioglu, G., Levitt, J. A., and Suhling, K. J. (2008). Molecular rotor measures viscosity of live cells via fluorescence lifetime imaging. J. Am. Chem. Soc. 130, 6672-6673. doi: 10.1021/ja800570d

Lee, S. C., Heo, J., Woo, H. C., Lee, J. A., Seo, Y. H., Lee, C. L., et al. (2018). Fluorescent molecular rotors for viscosity sensors. Chem. Eur. J. 24, 13706-13718. doi: 10.1002/chem.201801389

Li, J., Zhang, Y., Zhang, H., Xuan, X., Xie, M., Xia, S., et al. (2016). Nucleoside-based ultrasensitive fluorescent probe for the dual-mode imaging of microviscosity in living cells. Anal. Chem. 88, 5554-5560. doi: 10.1021/acs.analchem.6b01395

Li, L. L., Li, K., Li, M. Y., Shi, L., Liu, Y. H., Zhang, H., et al. (2018). BODIPY-based two-photon fluorescent probe for real-time monitoring of lysosomal viscosity with fluorescence lifetime imaging microscopy. Anal. Chem. 90, 5873-5878. doi: 10.1021/acs.analchem.8b00590

López-Duarte, I., Vu, T. T., Izquierdo, M. A., Bull, J. A., and Kuimova, M. K. (2014). A molecular rotor for measuring viscosity in plasma membranes of live cells. Chem. Commun. 50, 5282-5284. doi: 10.1039/C3CC47530A

Loudet, A., and Burgess, K. (2007). BODIPY dyes and their derivatives: syntheses and spectroscopic properties. Chem. Rev. 107, 4891-4932. doi: $10.1021 / \mathrm{cr} 078381 \mathrm{n}$

Lu, H., Mack, J., Yang, Y., and Shen, Z. (2014). Structural modification strategies for the rational design of red/NIR region BODIPYs. Chem. Soc. Rev. 43, 4778-4823. doi: 10.1039/C4CS00030G

Miao, W., Feng, Y., Wu, Q., Sheng, W., Li, M., Liu, Q., et al. (2019). Phenanthro[b]-fused BODIPYs through tandem Suzuki and oxidative aromatic couplings: synthesis and photophysical properties. J. Org. Chem. 84, 9693-9704. doi: $10.1021 /$ acs.joc.9b01425

Minton, A. P. (2001). The influence of macromolecular crowding and macromolecula confinement on biochemical reactions in physiological media. J. Biol. Chem. 276, 10577-10580. doi: 10.1074/jbc.R100005200

Nadiv, O., Shinitzky, M., Manu, H., Hecht, D., Roberts, C., LeRoith, D., et al. (1994). Elevated protein tyrosine phosphatase activity and increased membrane viscosity are associated with impaired activation of the insulin receptor kinase in old rats. Biochem. J. 298, 443-450. doi: 10.1042/bj2980443

Ning, P., Dong, P., Geng, Q., Bai, L., Ding, Y., Tian, X., et al. (2017). A two-photon fluorescent probe for viscosity imaging in vivo. J. Mater. Chem. B 5, 2743-2749. doi: 10.1039/C7TB00136C

Peterson, J. A., Wijesooriya, C., Gehrmann, E. J., Mahoney, K. M., Goswami, P. P., Albright, T. R., et al. (2018). Family of BODIPY photocages cleaved by single photons of visible/near-infrared light. J. Am. Chem. Soc. 140, 7343-7346. doi: 10.1021/jacs.8b04040

Song, X., Li, N., Wang, C., and Xiao, Y. (2017). Targetable and fixable rotor for quantifying mitochondrial viscosity of living cells by fluorescence lifetime imaging. J. Mater. Chem. B 5, 360-368. doi: 10.1039/C6TB02524B
Turksoy, A., Yildiz, D., and Akkaya, E. U. (2019). Photosensitization and controlled photosensitization with BODIPY dyes. Coord. Chem. Rev. 379, 47-64. doi: 10.1016/j.ccr.2017.09.029

Uzhinov, B. M., Ivanov, V. L., and Melnikov, M. Y. (2011). Molecular rotors as luminescence sensors of local viscosity and viscous flow in solutions and organized systems. Russ. Chem. Rev. 80, 1179-1190. doi: 10.1070/RC2011v080n12ABEH004246

Wang, L., Xiao, Y., Tian, W., and Deng, L. (2013). Activatable rotor for quantifying lysosomal viscosity in living cells. J. Am. Chem. Soc. 135, 2903-2906. doi: 10.1021/ja311688g

Wang, Z., Cheng, C., Kang, Z., Miao, W., Liu, Q., Wang, H., et al. (2019). Organotrifluoroborate salts as complexation reagents for synthesizing BODIPY dyes containing both fluoride and an organo substituent at the boron center. J. Org. Chem. 84, 2732-2740. doi: 10.1021/acs.joc. $8 \mathrm{~b} 03145$

Wu, Y., Štefl, M., Olzyńska, A., Hof, M., Yahioglu, G., Yip, P., et al. (2013). Molecular rheometry: direct determination of viscosity in $\mathrm{L}_{\mathrm{o}}$ and $\mathrm{L}_{\mathrm{d}}$ lipid phases via fluorescence lifetime imaging. Phys. Chem. Chem. Phys. 15, 14986-14993. doi: 10.1039/c3cp51953h

Yang, Z., He, Y., Lee, J. H., Chae, W. S., Ren, W. X., Lee, J. H., et al. (2014). A Nile Red/BODIPY-based bimodal probe sensitive to changes in the micropolarity and microviscosity of the endoplasmic reticulum. Chem. Commun. 50, 11672-11675. doi: 10.1039/C4CC04915B

Yang, Z., He, Y., Lee, J. H., Park, N., Suh, M., Chae, W. S., et al. (2013). A selfcalibrating bipartite viscosity sensor for mitochondria. J. Am. Chem. Soc. 135, 9181-9185. doi: 10.1021/ja403851p

Yu, C., Huang, Z., Gu, W., Wu, Q., Hao, E., Xiao, Y., et al. (2019). Uncommon meso-2-ketopyrrolylBODIPYs with AIE-active features for real-time quantitative viscosity mapping via fluorescence lifetime imaging microscopy. Mater. Chem. Front. 3, 1823-1832. doi: 10.1039/C9QM $00154 \mathrm{~A}$

Yu, H., Xiao, Y., and Jin, L. (2012). A lysosome-targetable and two-photon fluorescent probe for monitoring endogenous and exogenous nitric oxide in living cells. J. Am. Chem. Soc. 134, 17486-17489. doi: 10.1021/ja308967u

Zatsikha, Y. V., Didukh, N. O., Swedin, R. K., Yakubovskyi, V. P., Blesener, T. S., Healy, A. T., et al. (2019). Preparation of viscositysensitive isoxazoline/isoxazolyl-based molecular rotors and directly linked BODIPY-fulleroisoxazoline from the stable meso-(nitrile oxide)substituted BODIPY. Org. Lett. 21, 5713-5718. doi: 10.1021/acs.orglett. $9 \mathrm{~b} 02082$

Zhang, W., Sheng, W., Yu, C., Wei, Y., Wang, H., Hao, E., et al. (2017). Onepot synthesis and properties of well-defined butadiynylene-BODIPY oligomers. Chem. Commun. 53, 5318-5321. doi: 10.1039/C7CC02393F

Zhang, X., Sun, Q., Huang, Z., Huang, L., and Xiao, Y. (2019). Immobilizable fluorescent probes for monitoring the mitochondria microenvironment: a next step from the classic. J. Mater. Chem. B 7, 2749-2758. doi: 10.1039/C9TB00043G

Zhu, H., Fan, J., Li, M., Cao, J., Wang, J., and Peng, X. (2014). A “distortedBODIPY" -based fluorescent probe for imaging of cellular viscosity in live cells. Chem. Eur. J. 20, 4691-4696. doi: 10.1002/chem.201304296

Conflict of Interest: The authors declare that the research was conducted in the absence of any commercial or financial relationships that could be construed as a potential conflict of interest.

Copyright (C) 2019 Miao, Yu, Hao and Jiao. This is an open-access article distributed under the terms of the Creative Commons Attribution License (CC BY). The use, distribution or reproduction in other forums is permitted, provided the original author(s) and the copyright owner(s) are credited and that the original publication in this journal is cited, in accordance with accepted academic practice. No use, distribution or reproduction is permitted which does not comply with these terms. 